\title{
Moving Forward by Traveling in Circles
}

\author{
Stuart Boersma \\ Central Washington University
}

\section{Introduction}

The purpose of this paper is to introduce the reader to the mathematical construct known as holonomy. Holonomy is a measurement of the change in a certain angle as one travels along a curve. For this paper, we will consider two physical situations which involve "traveling in a circle" and comparing an initial and final measurement of an angle. In the first case we will see how this angular displacement can be used to prove that the Earth rotates! In the second example we explore the workings of a nineteenth century cartographic instrument. In both cases, traveling in circles yields interesting mathematical information.

\section{Foucault's Pendulum}

In the middle of the nineteenth century Foucault constructed a pendulum by hanging a $5 \mathrm{~kg}$ bob from the ceiling of a cellar (about $2 \mathrm{~m}$ high). After setting the pendulum in motion he observed "The oscillating point is continuously displaced..., which indicates that the deviation of the plane of oscillation takes place in the same sense as the apparent motion of the celestial sphere..." [1] Later on at the Pantheon he repeated this experiment on a grander scale using a $28 \mathrm{~kg}$ bob hanging from a height of $67 \mathrm{~m}$. Hopefully you have had the opportunity to observe this phenomenon yourself. If not, find such a pendulum nearby (science museums or large universities may have one) and organize a fieldtrip! Can you explain why he observed the plane of oscillation (the direction of the pendulum swing) rotating as time when on?

A couple of simple thought experiments can help replicate this phenomenon if you have never observed it firsthand. First, imagine a pendulum suspended from a 
great height directly above the North pole. Give the pendulum a slight push so that as it swings it almost brushes the ground. Now, assuming that it does not loose any momentum, imagine the pendulum swinging back and forth for a 24-hour period. What happens? Clearly the Earth rotates through a angle of $2 \pi$ so that an observer standing near the pendulum will observe the direction of swing of the pendulum steadily rotating through an angle of $2 \pi$ as well (over the same time period).

Now, perform the same thought experiment but this time let's suspend the pendulum directly over a point on the equator (say Quito). Suppose the pendulum is given a slight initial push so that it swings North-South. As the Earth rotates (Westto-East) the direction of the pendulum remains North-South! That is, there is no observable rotation of the plane of oscillation! Obviously there is something different about being at the North pole and the equator.

The next logical step would be to perform the thought experiment once again for some latitude between the equator and the North pole. However, at this point it is not as easy to keep track of the position of the pendulum relative to that of the surface of the Earth (try it!). However, one should be able to invoke a continuity argument to claim that since the pendulum does not rotate when positioned at the equator and it rotates through an angle of $2 \pi$ when positioned at the North pole (over a 24-hour period), it must continuously rotate through greater and greater angles as one positions it in more northern climes. Nevertheless, it is the nature of the increase which is the more interesting question. Is this increase linear? Quadratic? Something else?

We will describe the phenomenon of Foucault's pendulum mathematically. Without relying on any incantations of such physical quantities as "coriolis effect" and the like, we will show that the phenomenon is just a simple effect involving the curvature of the earth and the notion of "parallel" on a surface; two ingredients for the study of holonomy! 


\section{Life On a Sphere}

Since you are currently reading a mathematics journal, you are no doubt aware that there are some fundamental differences between a sphere and a plane. For instance,

- In a plane, all triangles have angles which sum to exactly $180^{\circ}$ while on the surface of a sphere triangles have angles which sum to greater than $180^{\circ}$.

- In a plane, parallel lines never meet. On a sphere they meet twice! (Just imagine two travelers starting nearby on the equator. If they decide to each walk North they will meet at the North pole and again at the South pole as "lines" on spheres are simply great circles.)

These two observations indicate that geometry on a sphere is certainly different then geometry on a plane. However, there are also topological differences as well.

- Imagine a group of people standing in a circle and holding hands. If they were standing on a plane and began to walk "towards" each other the circle would grow increasingly smaller until everyone met at a point. Or, if they walked away from each other the circle would continue to grow larger and larger (and everyone's arms would have to stretch indefinitely!). Now, if this same circle of people were standing on the surface of a sphere they could begin to walk either towards one another or away from one another and eventually they would still meet. (It might take a while and it would require a lot of arm stretching, but it could be done!)

- More simply, just imagine a lone world-traveler always waking in a straight line. In a plane this traveler would keep walking forever without ever passing over the same ground twice. However, on a sphere the traveler would eventually return to her starting point.

Why do these differences exist? Because of CURVATURE! Curvature affects geometry (triangles, notions of parallel) as well as physics (paths that light travel, 
Einstein's theory of general relativity, and Foucault's pendulum which we are still getting to). In order to see the effect of curvature on Foucault's pendulum we will first have to learn how to do calculus and geometry on a sphere.

\section{Doing Calculus on a Sphere}

Just as in any mathematics course, one needs to be equipped with the appropriate tools in order to correctly do geometry and calculus on a sphere. For our purposes here, we will need the following tools: appropriate coordinates, basis vectors that are related to our chosen coordinates, and an appropriate notion of derivative.

\section{Coordinates and Basis Vectors}

Since the Earth is a sphere (basically) it probably makes the most sense to work in spherical coordinates. Most readers are probably familiar with these coordinates $(\rho, \theta$, and $\phi)$, yet fewer are probably familiar with the corresponding basis vectors which are so crucially linked to these coordinates $(\overrightarrow{\boldsymbol{\rho}}, \overrightarrow{\boldsymbol{\theta}}, \overrightarrow{\boldsymbol{\phi}})$. These basis vectors are defined in an analogous way as the cartesian basis vectors $\overrightarrow{\boldsymbol{\imath}}, \overrightarrow{\boldsymbol{\jmath}}$, and $\overrightarrow{\boldsymbol{k}}$. Specifically,

- $\overrightarrow{\boldsymbol{\rho}}$ is the unit vector which points in the direction of increasing $\rho$ (the radial coordinate),

- $\overrightarrow{\boldsymbol{\theta}}$ is the unit vector which points in the direction of increasing $\theta$ (the angle from the positive $x$-axis in the $x y$-plane), and

- $\vec{\phi}$ is the unit vector which points in the direction of increase $\phi$ (the angle measured down from the positive $z$-axis).

For example, consider a point $P$ with spherical coordinates $(1,0, \pi / 2)$ located on the equator of a sphere of radius one. At $P, \overrightarrow{\boldsymbol{\rho}}$ is perpendicular to the sphere (the radial direction is perpendicular to the surface of the sphere), $\overrightarrow{\boldsymbol{\theta}}$ points East (and is tangent to the sphere), and $\vec{\phi}$ points South (and is also tangent to the sphere). Compare the directions of these vectors with those based at point $Q$ with coordinates $(1, \pi / 4, \pi / 4)$ (see Figure 1). 


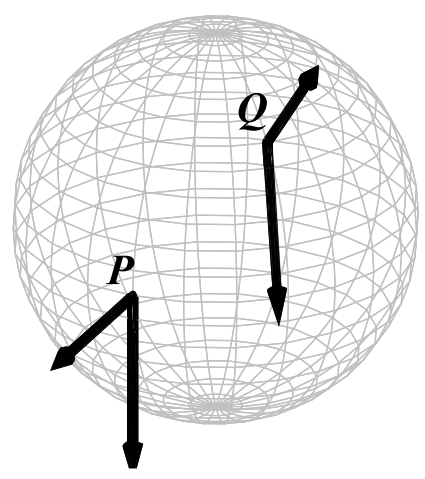

Figure 1: Spherical basis vectors $\vec{\rho}$ and $\vec{\phi}$ at two different points on a sphere.

Experiment with visualizing $\overrightarrow{\boldsymbol{\rho}}, \overrightarrow{\boldsymbol{\theta}}$, and $\overrightarrow{\boldsymbol{\phi}}$ at other points on the sphere. There are a few similarities and a few differences between spherical coordinate vectors and rectangular coordinate vectors. The similarities include the facts that they are all of unit length (by definition) and $\overrightarrow{\boldsymbol{\rho}}, \overrightarrow{\boldsymbol{\theta}}$, and $\overrightarrow{\boldsymbol{\phi}}$ are all mutually perpendicular. Thus, they form a very nice basis for $\mathbb{R}^{3}$. The most intriguing difference stems from the fact that these vectors "change direction" as one moves about $\mathbb{R}^{3}$. That is, the vector (directed line segment) $\overrightarrow{\boldsymbol{\rho}}$ at $P$ points in a different direction from the vector (directed line segment) $\overrightarrow{\boldsymbol{\rho}}$ at $Q$ even though they both represent $\overrightarrow{\boldsymbol{\rho}}$ and they both point in the direction of increasing $\rho$ at their respective base points.

Since we are interested in doing calculus on Earth, we need to understand how these vectors change directions as their "base points" move around space. Actually, for the pendulum problem we are simply interested in points as they move around Earth, a sphere of fixed radius. Mathematically, we wish to compute the rates of change of the basis vectors $\overrightarrow{\boldsymbol{\rho}}, \overrightarrow{\boldsymbol{\theta}}$, and $\overrightarrow{\boldsymbol{\phi}}$ with respect to the coordinates $\theta$ and $\phi$. We will proceed by geometrically analyzing a small displacement of a specific basis vector as one of the coordinates increases by a slight amount.

\section{Analyzing $\overrightarrow{\boldsymbol{\rho}}$}

Compare the radial basis vectors $\overrightarrow{\boldsymbol{\rho}}$ at nearby points $(1, \theta, \phi)$ and $(1, \theta+d \theta, \phi)$. To help with this comparison, imagine each of these radial unit vectors with their base 
points at the origin (see Figure 2).
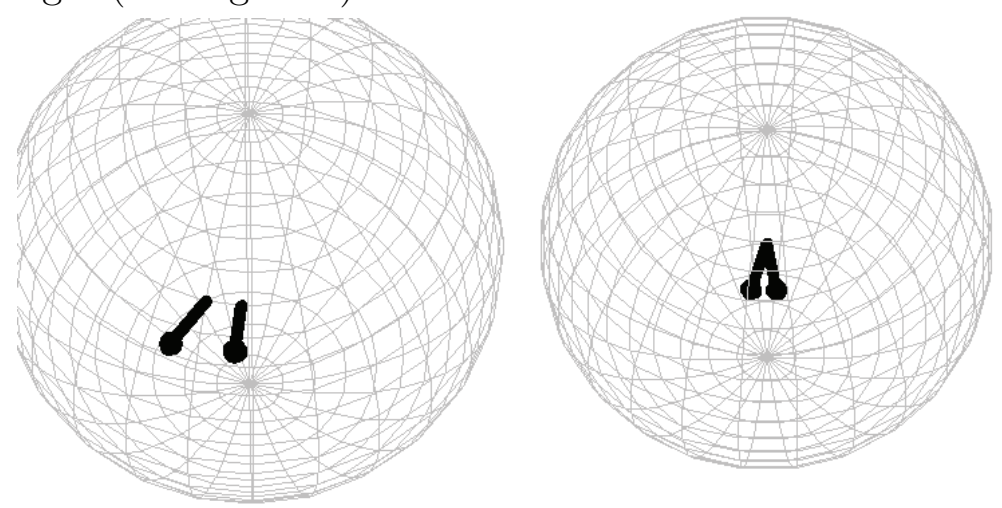

Figure 2: The lefthand figure shows $\overrightarrow{\boldsymbol{\rho}}$ at two nearby points. The righthand figure shows the same vectors translated to the origin.

Since they represent, respectively, $\overrightarrow{\boldsymbol{\rho}}$ at $(1, \theta, \phi)$ and $(1, \theta+d \theta, \phi)$, they will be pointing in different directions. Use $d \overrightarrow{\boldsymbol{\rho}}$ to represent this small vector displacement. Since the motion was generated by a small angular displacement of $d \theta$ in the $x y$-plane, the length of $d \overrightarrow{\boldsymbol{\rho}}$ is governed by the length of the projection of $\overrightarrow{\boldsymbol{\rho}}$ into the $x y$-plane. Thus the length of $d \overrightarrow{\boldsymbol{\rho}}$ is $\sin \phi d \theta$ (see Figure 3).

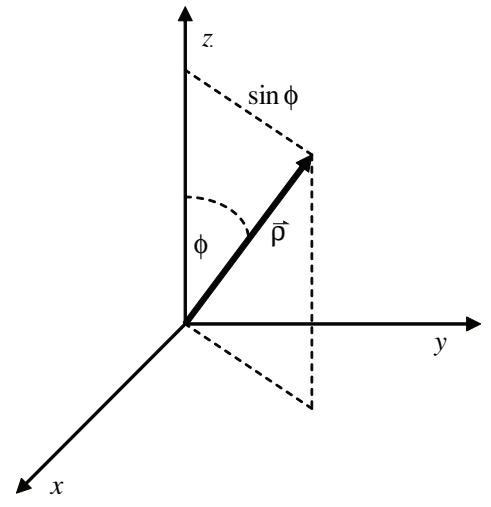

Figure 3: The basis vector $\overrightarrow{\boldsymbol{\rho}}$ projected down to the $x y$-plane has length $\sin \phi$.

Additionally, $d \overrightarrow{\boldsymbol{\rho}}$ points in the direction of increasing $\theta$. Thus, keeping $\rho$ and $\phi$ fixed and varying $\theta$ by $d \theta$ we have $d \overrightarrow{\boldsymbol{\rho}}=\sin \phi d \theta \overrightarrow{\boldsymbol{\theta}}$. "Dividing" by $d \theta$ yields our first rate of change that we are interested in. Using partial derivative notation we have

$$
\frac{\partial \overrightarrow{\boldsymbol{\rho}}}{\partial \theta}=\sin \phi \overrightarrow{\boldsymbol{\theta}}
$$


As a homework assignment, try to argue that

$$
\frac{\partial \overrightarrow{\boldsymbol{\rho}}}{\partial \phi}=\vec{\phi}
$$

\section{Analyzing $\vec{\phi}$}

Once again, compare $\vec{\phi}$ at $(1, \theta, \phi)$ and $(1, \theta+d \theta, \phi)$ by translating these vectors to the origin and letting $d \vec{\phi}$ represent the displacement vector. The motion is very similar to that of $\overrightarrow{\boldsymbol{\rho}}$ above. However, this time the projected length of $\overrightarrow{\boldsymbol{\phi}}$ into the $x y$-plane is $\cos \phi$ (see Figure 4 ). (Note that the $\phi$ coordinate of $\vec{\phi}$ also measures the angle made by $\vec{\phi}$ and the $x y$-plane.)

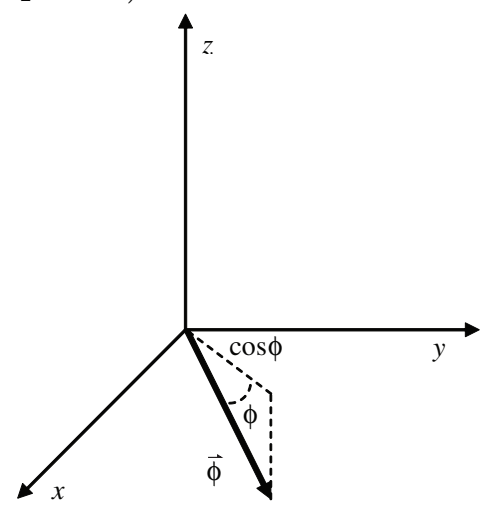

Figure 4: The basis vector $\vec{\phi}$ projected to the $x y$-plane has length $\cos \phi$.

The direction of motion is as before yielding $d \overrightarrow{\boldsymbol{\phi}}=\cos \phi d \theta \overrightarrow{\boldsymbol{\theta}}$. This gives rise to our third rate of change

$$
\frac{\partial \vec{\phi}}{\partial \theta}=\cos \phi \overrightarrow{\boldsymbol{\theta}}
$$

For homework show that

$$
\frac{\partial \vec{\phi}}{\partial \phi}=-\overrightarrow{\boldsymbol{\rho}}
$$

\section{Analyzing $\overrightarrow{\boldsymbol{\theta}}$}

We will proceed as before by holding both $\rho$ and $\phi$ fixed, increasing or decreasing $\theta$ by $d \theta$, and translating $\overrightarrow{\boldsymbol{\theta}}$ at nearby points to the origin. Much like motion around a circle, $d \overrightarrow{\boldsymbol{\theta}}$ will directed in towards the center of a circle in a plane parallel to the $x y$-plane (see Figure 5). 


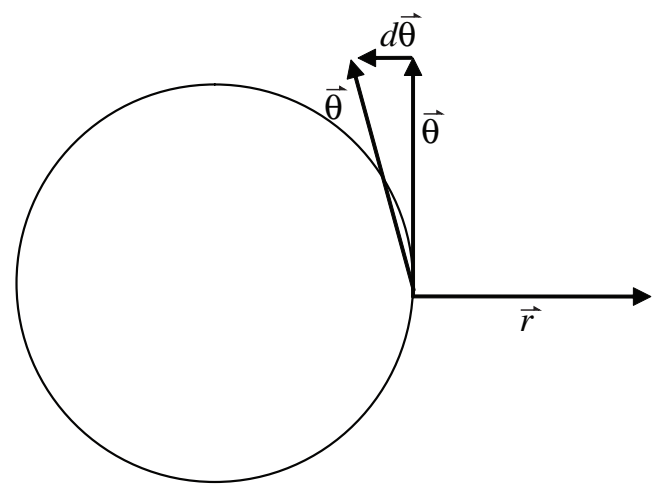

Figure 5: In a plane parallel to the $x y$-plane, one sees that increasing the coordinate $\theta$ creates a displacement $d \overrightarrow{\boldsymbol{\theta}}$ of $\overrightarrow{\boldsymbol{\theta}}$. This displacement is in the direction of $-\overrightarrow{\boldsymbol{r}}$.

If $\overrightarrow{\boldsymbol{r}}$ represents the unit vector in the direction of the cylindrical coordinate $r$, one would have $d \overrightarrow{\boldsymbol{\theta}}=-d \theta \overrightarrow{\boldsymbol{r}}$. However, we need to express $\overrightarrow{\boldsymbol{r}}$ in terms of our basis vectors $\overrightarrow{\boldsymbol{\rho}}, \overrightarrow{\boldsymbol{\theta}}$, and $\overrightarrow{\boldsymbol{\phi}}$. Looking at the projections of $\overrightarrow{\boldsymbol{r}}$ onto $\overrightarrow{\boldsymbol{\rho}}$ and $\overrightarrow{\boldsymbol{\phi}}$ yield $\overrightarrow{\boldsymbol{r}}=\sin \phi \overrightarrow{\boldsymbol{\rho}}+\cos \phi \overrightarrow{\boldsymbol{\phi}}$ (see Figure 6).

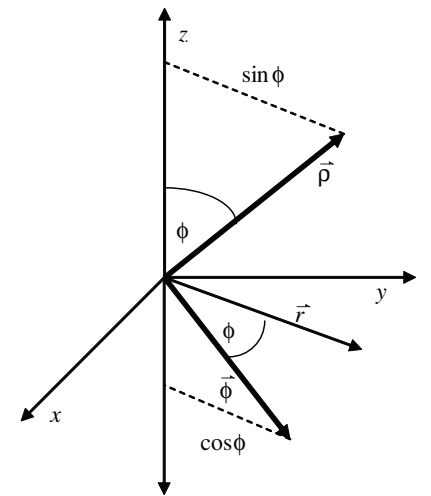

Figure 6: At point $(\rho, \theta, \phi), \overrightarrow{\boldsymbol{\rho}}$ makes an angle of $\phi$ with the positive $z$-axis and $\overrightarrow{\boldsymbol{\phi}}$ makes an angle of $\phi$ with the $x y$-plane. The radial vector in the $x y$-plane can now be written $\overrightarrow{\boldsymbol{r}}=\sin \phi \overrightarrow{\boldsymbol{\rho}}+\cos \phi \overrightarrow{\boldsymbol{\phi}}$.

Note that $\overrightarrow{\boldsymbol{r}}$ is perpendicular to $\overrightarrow{\boldsymbol{\theta}}$ and has no component in that direction. Thus, $d \overrightarrow{\boldsymbol{\theta}}=-\sin \phi d \theta \overrightarrow{\boldsymbol{\rho}}-\cos \phi d \theta \overrightarrow{\boldsymbol{\phi}}$ and

$$
\frac{\partial \overrightarrow{\boldsymbol{\theta}}}{\partial \theta}=-\sin \phi \overrightarrow{\boldsymbol{\rho}}-\cos \phi \overrightarrow{\boldsymbol{\phi}}
$$

The final homework assignment is to show that

$$
\frac{\partial \overrightarrow{\boldsymbol{\theta}}}{\partial \phi}=0 .
$$




\section{The Pendulum}

Armed with the necessary tools, we now come back to the original study of the pendulum. As we begin to analyze this motion, keep three important facts in mind:

1. The pendulum swings close to the surface of the sphere through a very small angle and, hence, the direction of swing, which we will call $\overrightarrow{\boldsymbol{v}}$, is tangent to the sphere.

2. The pendulum itself is just trying to go "back and forth"; it is not trying to twist or rotate. It is the Earth under the pendulum which is rotating! As the Earth rotates, we can think of the pendulum traveling along a circle of constant latitude $\left(\phi=\phi_{0}\right)$.

3. No special attention to physical forces will be given. Remember we are treating this as a geometry problem not a physics problem.

Given these assumptions, we will cast the phenomenon of Foucault's pendulum into mathematical terms.

\section{The Geometry Problem:}

Given a unit vector $\overrightarrow{\boldsymbol{v}}$, "slide" it around a circle of constant latitude such that

1. $\overrightarrow{\boldsymbol{v}}$ is always tangent to the sphere (which means that $\overrightarrow{\boldsymbol{v}}$ can always be expressed as a linear combination of $\overrightarrow{\boldsymbol{\theta}}$ and $\overrightarrow{\boldsymbol{\phi}})$, and

2. The direction of $\overrightarrow{\boldsymbol{v}}$ "always stays the same". That is, $\overrightarrow{\boldsymbol{v}}$ does not change in the $\overrightarrow{\boldsymbol{\theta}}$ and $\vec{\phi}$ directions (the only directions that we care about!).

The above problem is a common one in differential geometry. Using fancy language we want to "parallel transport" $\overrightarrow{\boldsymbol{v}}$ around a circle of constant latitude. We can then compare what $\overrightarrow{\boldsymbol{v}}$ looks like after we make a complete trip around the Earth (i.e. after 24 hours have elapsed).

Now, as $\overrightarrow{\boldsymbol{v}}$ travels around a circle of constant latitude, only $\theta$ changes. Hence we can think of $\overrightarrow{\boldsymbol{v}}$ (the direction of swing) as being parameterized by $\theta$. Furthermore, we are assuming that the pendulum's swing is always tangent to the sphere and hence 
can be written as a linear combination of $\overrightarrow{\boldsymbol{\theta}}$ and $\overrightarrow{\boldsymbol{\phi}}$. We have,

$$
\overrightarrow{\boldsymbol{v}}(\theta)=a(\theta) \overrightarrow{\boldsymbol{\theta}}+b(\theta) \overrightarrow{\boldsymbol{\phi}}
$$

where $a$ and $b$ are scalars that depend on $\theta$. To see how $\overrightarrow{\boldsymbol{v}}$ changes as it travels around a circle of constant latitude (increasing $\theta$ ) we need to compute $\frac{\partial}{\partial \theta} \overrightarrow{\boldsymbol{v}}$. Additionally, since we are only interested in motion tangent to the sphere we will project this partial derivative down to the surface of the sphere. Thus, as we start taking derivatives, just keep in mind that any component in the $\overrightarrow{\boldsymbol{\rho}}$ direction will be ignored and we'll only keep those components in the directions of $\overrightarrow{\boldsymbol{\theta}}$ and $\overrightarrow{\boldsymbol{\phi}}$. Furthermore, use $\nabla \overrightarrow{\boldsymbol{v}}$ to represent $\frac{\partial}{\partial \theta} \overrightarrow{\boldsymbol{v}}$ projected down to the sphere. Thus,

$$
\begin{aligned}
\nabla \overrightarrow{\boldsymbol{v}} & =\nabla(a(\theta) \overrightarrow{\boldsymbol{\theta}}+b(\theta) \overrightarrow{\boldsymbol{\phi}}) \\
& =a^{\prime} \overrightarrow{\boldsymbol{\theta}}+a\left(-\cos \phi_{0} \overrightarrow{\boldsymbol{\phi}}\right)+b^{\prime} \overrightarrow{\boldsymbol{\phi}}+b\left(\cos \phi_{0} \overrightarrow{\boldsymbol{\theta}}\right) \\
& =\left(a^{\prime}+b \cos \phi_{0}\right) \overrightarrow{\boldsymbol{\theta}}+\left(b^{\prime}-a \cos \phi_{0}\right) \overrightarrow{\boldsymbol{\phi}} .
\end{aligned}
$$

Since we are stipulating that $\nabla \overrightarrow{\boldsymbol{v}}=\overrightarrow{\mathbf{0}}$, this will only be the case when

$$
a^{\prime}+b \cos \phi_{0}=0
$$

and

$$
b^{\prime}-a \cos \phi_{0}=0
$$

Which means we have a system of differential equations:

$$
\begin{aligned}
& \frac{d a}{d \theta}=-b \cos \phi_{0} \\
& \frac{d b}{d \theta}=a \cos \phi_{0} .
\end{aligned}
$$

We can solve this uniquely once we impose initial conditions. If we assume that the pendulum is started so that it swings east-west (in the $\theta$ direction only) we have $\overrightarrow{\boldsymbol{v}}(0)=1 \overrightarrow{\boldsymbol{\theta}}+0 \overrightarrow{\boldsymbol{\phi}}$ which gives us the initial conditions of $a(0)=1$ and $b(0)=0$. To simplify the results, let $Q=\cos \phi_{0}$. Even if you have never had a course in differential equations, you can probably guess what the solution to the above initial value problem is. Think about it. We are looking for two functions of $\theta$. The first one, 
$a(\theta)$, has a derivative which is a multiple the second one, $b(\theta)$, while the derivative of the second function is a multiple of the first! The basic trigonometric functions should quickly come to mind. Together with the initial conditions, one sees that the unknown functions $a$ and $b$ must be

$$
\begin{aligned}
& a(\theta)=\cos Q \theta \\
& b(\theta)=\sin Q \theta .
\end{aligned}
$$

Recall that since the direction of the pendulum's swing is given by $\overrightarrow{\boldsymbol{v}}=a(\theta) \overrightarrow{\boldsymbol{\theta}}+b(\theta) \overrightarrow{\boldsymbol{\phi}}$, we now know how the direction of the swing moves as the Earth rotates ( $\theta$ increases)! For a more geometrical interpretation of how $\overrightarrow{\boldsymbol{v}}$ changes, exploit the power of matrix multiplication to write

$$
\left[\begin{array}{l}
a(\theta) \\
b(\theta)
\end{array}\right]=\left[\begin{array}{cc}
\cos Q \theta & -\sin Q \theta \\
\sin Q \theta & \cos Q \theta
\end{array}\right]\left[\begin{array}{l}
1 \\
0
\end{array}\right]
$$

This matrix multiplication may be familiar from linear algebra. The vector $[a(\theta), b(\theta)]$ is simply the vector $[1,0]$ after having been rotated through an angle of $Q \theta$. On Earth, we typically describe positions with latitude as opposed to the spherical coordinate $\phi$. If $L$ represents one's latitude we have

$$
\begin{aligned}
Q & =\cos \phi_{0} \\
& =\cos (\pi / 2-L) \\
& =\sin (L) .
\end{aligned}
$$

So, after 24 hours of swinging, the pendulum (i.e. $\overrightarrow{\boldsymbol{v}}$ ) would end up rotating by $2 \pi Q$ where $Q$ is the sine of your latitude! A quick reality check shows that this result agrees with our earlier thought experiments. At the equator $(L=0)$ there is no oscillation of the pendulum, while at the North pole $(L=\pi / 2)$ the pendulum completes exactly the same rotation as the Earth! 


\section{A New Instrument}

In 1886 a paper publicized a new instrument to measure the area of closed plane curves. The paper was authored by a Dane identified simply as "Z" [2]. The instrument looked roughly like a square bracket "[" in which one of the bracket's "points" was indeed a pointed tip while the other consisted of short knife edge. These two ends were connected by a central metallic rod (see Figure 7) and the instrument was known for a while as a "stang" planimeter (stang is Danish for rod).

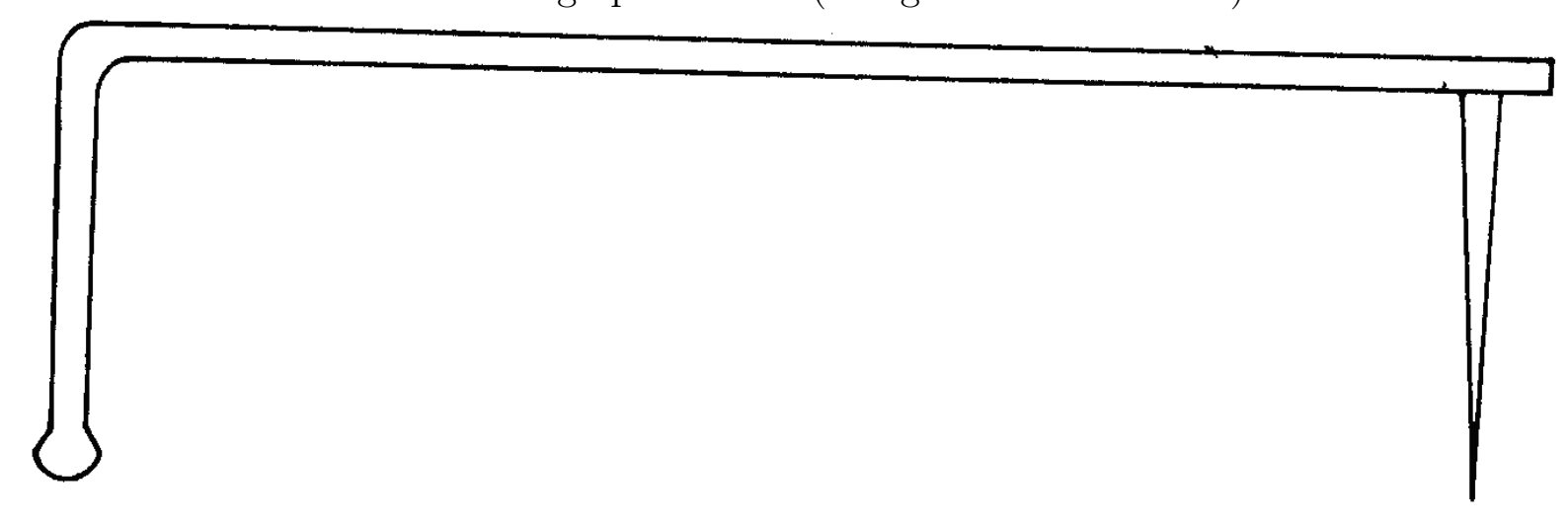

Figure 7: A Prytz planimeter with the tracing tip on the right and the knife edge on the left.

It has also been referred to as a "hatchet planimeter", "hacksaw blade planimeter", or the "Prytz planimeter" once it became known that "Z" was Holger Prytz.

To use the Prytz planimeter to measure the area enclosed by a closed planar curve (most likely a region depicted on a map), one would use the tip of the planimeter to trace around the curve while the knife edge of the planimeter followed the tip in a manner similar to the rear wheel of a bicycle following the front wheel as the bicycle meanders along. The knife edge serves a special purpose. Before one begins tracing the curve, press down on the knife edge leaving a noticeable indentation on the surface. After tracing the entire curve so the tip returns to its starting point, it turns out that the rod will not be in its original orientation. By pressing down a second time, another indentation is created. Both indentations are equidistant from the starting point on the curve, yet one could rotate the final position of the 
knife edge onto its original position). Let $s$ represent the arc length between the two indentations (see Figure 8 ) and $L$ the length of the planimeter's rod. Prytz, writing as "Z", claimed that the area bounded by the closed curve could be approximated by the simple product $L \cdot s$.

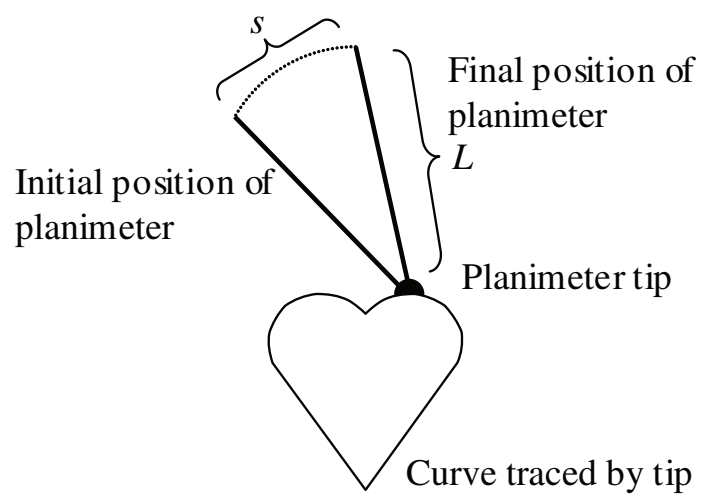

Figure 8: The initial and final position of the planimeter are separated by an arc of length $s$.

There are similarities between this process and that of Foucault's pendulum. In the case of the pendulum we saw that the orientation of the plane of the swinging bob changed orientation after it (or the Earth) had completed a trip around a closed curve of constant latitude. In the case of the Prytz planimeter, the rod changes orientation as the tip completes a trip around a closed curve. In both cases the change in orientation measures an interesting geometric property. In the case of the pendulum one measures the latitude of the location of the pendulum, in the case of the Prytz planimeter one measures the area enclosed by the curve. As mentioned before, these are both examples of a mathematical phenomenon known as holonomy.

\section{Pursuit Curves}

Before trying to understand how the Prytz planimeter measures area, we should look at a few examples of how the knife edge follows the tracing tip. The simplest case is probably that in which the curve, $\gamma$, is a straight line segment. Let $\gamma$ represent a straight horizontal line segment. Place the tip of the planimeter at the lefthand endpoint of $\gamma$ and orient the rod so that it is perpendicular to $\gamma$. As the tip is moved 
down the line to the right, the knife edge will always be pointed towards the tip and will begin to move closer to $\gamma$. The curve which the knife edge traces out is known as a pursuit curve (think of a rabbit running along $\gamma$ as a fox chases it by always running towards the current location of the rabbit). (See Figure 9 for a brief animation.) The curve in this case is also known as the "tractrix".

\section{Figure 9: Animation of a Prytz planimeter tracing a straight line.}

Now that one has developed a bit of intuition regarding the motion of the knife edge, consider the case where $\gamma$ represents a rectangle. Let $\gamma$ be a rectangle of width two and height one with the origin at the lower lefthand corner. Begin with the planimeter's tip at the origin, the knife edge at the point $(0,5)$, and trace $\gamma$ counterclockwise. As the tip traces the first side of the rectangle the knife edge will follow a tractrix as before. Before looking ahead, try to figure out the motion of the knife edge as one continues around the rectangle. Figure 10 shows an animation of the motion of the planimeter as well as the curve which the edge traces. Note that even though the tip returns to the starting point, the curve the knife edge traces out is not closed.

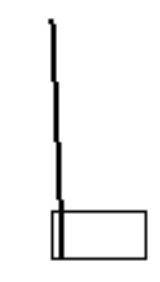

Figure 10: Animation of a Prytz planimeter tracing a rectangle.

For a third example, let's trace around a circle. Figure 11 shows a planimeter of length five traversing a circle of radius one. 


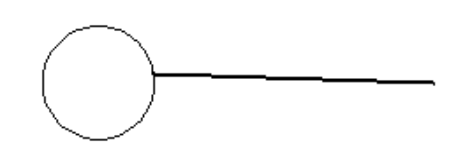

Figure 11: Follow link for an animation of a Prytz planimeter tracing a circle.

Except for the case where $\gamma$ was a straight line, the motion of knife edge is very difficult to predict and the crazy zig-zag curve which it follows appears to bear no geometric relationship to the curve the tip is tracing.

\section{Further Investigations}

Figure 12 shows the initial and final position of the planimeter for the case of the $2 \times 1$ rectangle from above.

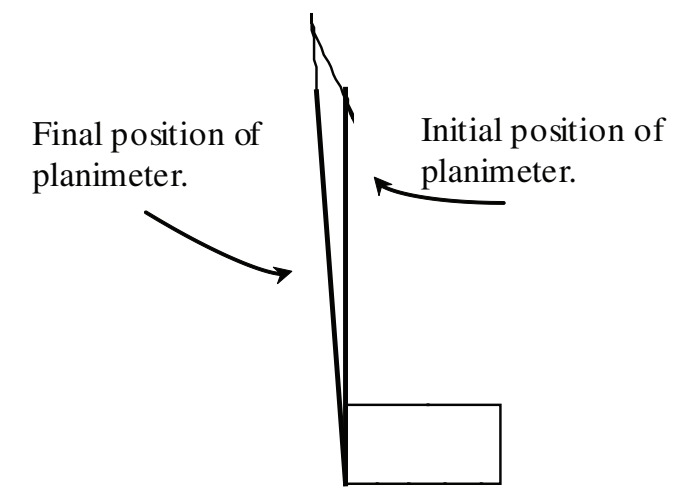

Figure 12: Initial and final position of planimeter $(L=5)$ after tracing the rectangle in a counter-clockwise direction.

Earlier it was claimed that the area of the rectangle was related to the length of the planimeter and the arc length representing the distance between the original and final positions of the knife edge (measure the length of the arc generated by the knife edge of the planimeter as it is rotated about it's tip to bring the final position into alignment with the original position). In this example $L=5$ and it turns out that $s \approx .375$ yielding $L s=1.875$. But what if one used a shorter planimeter?

Figure 13 animates the motion around the same rectangle with a planimeter of length $L=1$. 


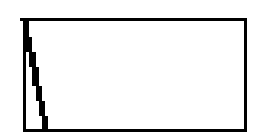

Figure 13: Follow link for an animation of the $L=1$ planimeter tracing the rectangle.

In this case the change in orientation of the knife edge is much greater with $s \approx 1.391$ yielding an estimate of the area as $L s=1.391$, much smaller than our first estimate (and the actual area). The initial and final positions of the planimeter are shown in Figure 14.

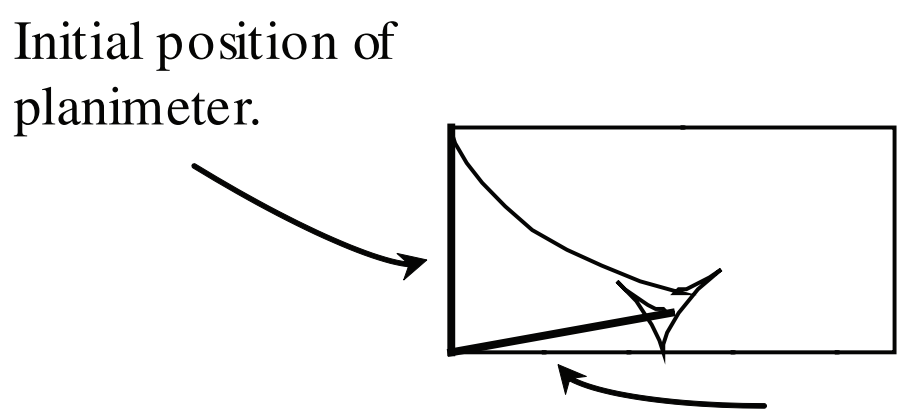

\section{Final position of planimeter.}

Figure 14: Initial and final position of planimeter $(L=1)$ after tracing the rectangle in a counter-clockwise direction.

Further complications arise when one changes the starting orientation of the planimeter. Using the same rectangle, Figure 15 shows the results of a planimeter of length $L=1$ which starts out pointing down the $x$-axis. In this case $s \approx .781$. Smaller yet.

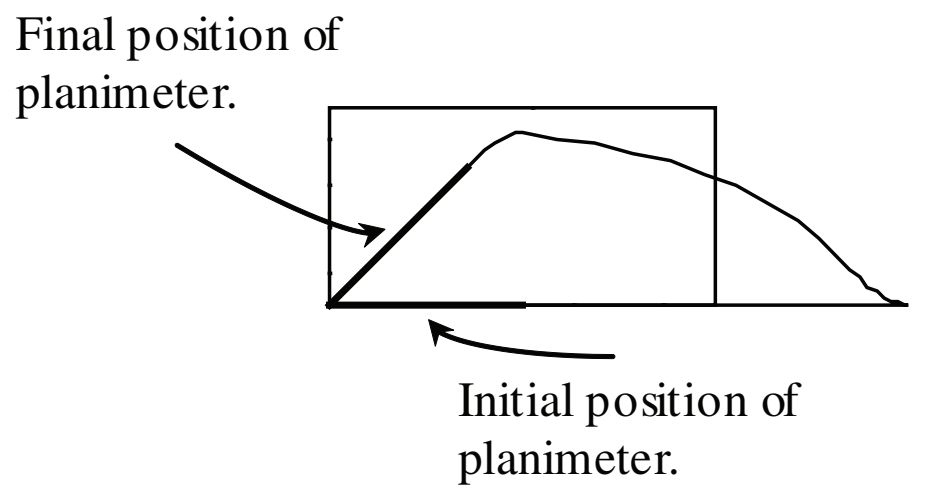

Figure 15: Initial and final position of planimeter $(L=1)$ after tracing the rectangle in a counter-clockwise direction. 
These investigations are a bit disconcerting as to the usefulness of this device for measuring area. Different planimeters will yield different estimates, identical planimeters will yield different estimates, and all of these estimates vary quite a bit from the true area! Let's turn to some mathematical analysis of the motion of the Prytz planimeter to help unravel some of these conundrums.

\section{Mathematical Analysis}

We will begin by studying the area swept out by a moving line segment. Consider a line segment $\overline{P Q}$ of length $L$ which is moved a "little bit" to the new location $\overline{P^{\prime} Q^{\prime}}$. This total motion can be decomposed into a translation $(\overline{P Q}$ is translated so that $P$ coincides with $P^{\prime}$ ) followed by a rotation (bringing $Q$ to $Q^{\prime}$ ). We will study each motion separately.

For the translation, consider $\overline{P Q}$, with unit normal vector $\vec{N}$, being translated to $\overline{P^{\prime} Q^{\prime \prime}}$. The area swept out by this motion, $d A_{1}$ corresponds to the area of the parallelogram $P Q Q^{\prime \prime} P^{\prime}$ (see Figure 16).

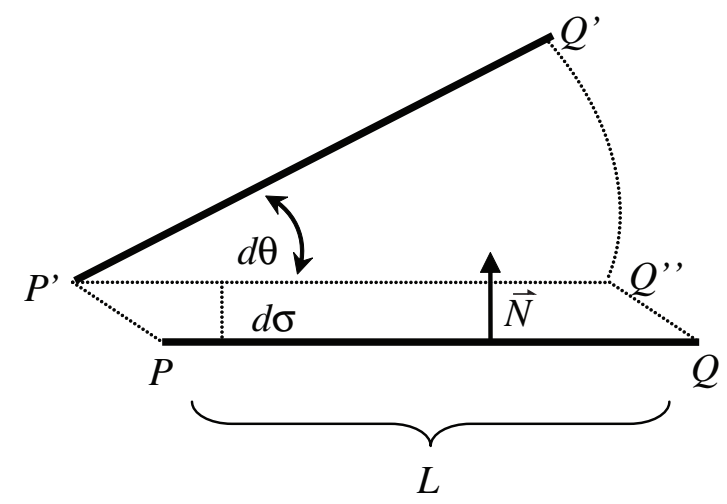

Figure 16: Line segment $\overline{P Q}$ moves to $\overline{P^{\prime} Q^{\prime}}$ via a translation followed by a rotation.

If we let $d \sigma$ represent the height of the parallelogram (which corresponds to the amount of motion perpendicular to $\overline{P Q}$ ), the area of the parallelogram is $L d \sigma$. Later we will be interested in signed area, so we will call this area positive if $\vec{N}$ points into the region and negative if $\overrightarrow{\boldsymbol{N}}$ points out of the region. Thus $d A_{1}= \pm L d \sigma$ with the sign determined by the choice of $\vec{N}$. 
The final total motion is now obtained by rotating $\overline{P^{\prime} Q^{\prime \prime}}$ about $P$ by an angle of $d \theta$ to result in $\overline{P^{\prime} Q^{\prime}}$. The area of this sector is $\frac{1}{2} L^{2} d \theta$ so we will define $d A_{2}= \pm \frac{1}{2} L^{2} d \theta$ where the sign is again determined by orientation of $\vec{N}$. Thus, the total area swept out by the line as it moves from $\overline{P Q}$ to $\overline{P^{\prime} Q^{\prime}}$ is

$$
\begin{aligned}
d A & =d A_{1}+d A_{2} \\
& =L d \sigma+\frac{1}{2} L^{2} d \theta .
\end{aligned}
$$

Now consider the line segment $\overline{P Q}$ moving in a very constrained way. Let $\overline{P Q}$ moves in such a way that $P$ and $Q$ both make a single circuit around simple closed curves $\gamma_{P}$ and $\gamma_{Q}$. In Figure 17 we see that the area being swept out by the line segment initially includes the region enclosed by $\gamma_{Q}$ (positive area) and a region between $\gamma_{P}$ and $\gamma_{Q}$ (also positive area).

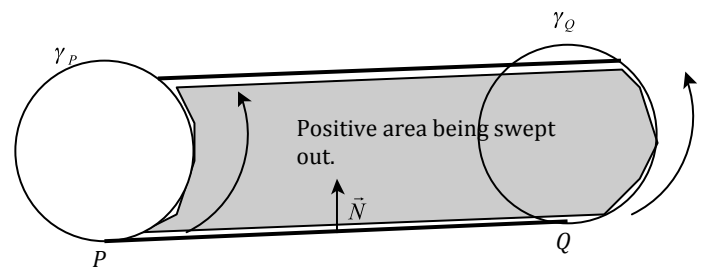

Figure 17: As $P$ and $Q$ begin to traverse the two curves counter-clockwise, the line segment connecting them sweeps out an area in the direction of $\vec{N}$.

In the later half of the motion (Figure 18), we see that the area being swept out includes the region enclosed by $\gamma_{P}$ (negative area) and the same region as before that lies between $\gamma_{P}$ and $\gamma_{Q}$ (this time we get negative area). 


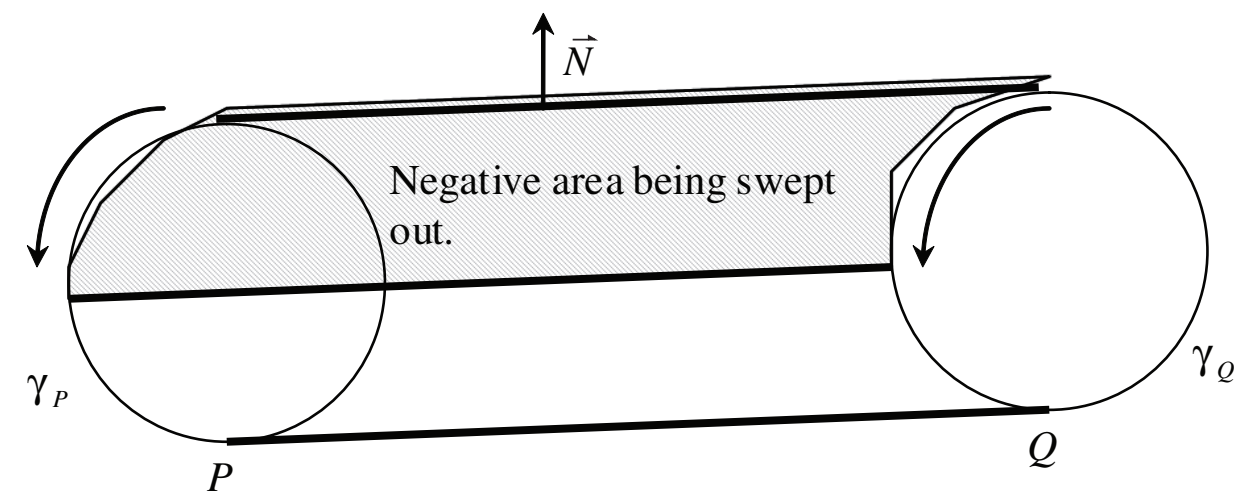

Figure 18: As the line segment returns to its starting position, negative area is swept out.

Thus, when $P$ and $Q$ return to their starting points we have

$$
A=A_{Q}-A_{P}
$$

where $A_{P}$ and $A_{Q}$ represent the areas enclosed by $\gamma_{P}$ and $\gamma_{Q}$ respectively.

To apply this analysis to the Prytz planimeter, let $Q$ represent the tracing tip and $P$ the knife edge. Thus, $\gamma_{Q}$ will be the closed curve that the planimeter tip traces. As noted before, when the tip completes a circuit of $\gamma_{Q}$, the knife edge $(P)$ traces out a curve $\gamma_{1 P}$ which is not closed! However, if we add the arc which connects the final and initial positions of $P$, denoted by $\gamma_{2 P}$, we will have a closed curve $\gamma_{P}$.

The total (net-signed) area swept out by $\overline{P Q}$ can be obtained by integrating $d A=L d \sigma+\frac{1}{2} L^{2} d \theta$. By design, as $Q$ traces $\gamma_{Q}$, the knife edge $P$ "follows" the tip $Q$. That is, there is no motion perpendicular to $\overline{P Q}$ ! (This is reminiscent of parallel transport in the case of the pendulum.) This implies that $d \sigma=0$ on $\gamma_{1 P}$. Also, after $P$ has traversed $\gamma_{1 P}$ as well as the $\operatorname{arc} \gamma_{2 P}$, the planimeter is in its original orientation. This means that

$$
\int_{\gamma_{P}} d \theta=0
$$


Thus, the total area swept out by $\overline{P Q}$ will be

$$
\begin{aligned}
A & =\int_{\gamma_{P}} d A \\
& =\int_{\gamma_{P}}\left(L d \sigma+\frac{1}{2} L^{2} d \theta\right) \\
& =\int_{\gamma_{P}} L d \sigma+0 \\
& =\int_{\gamma_{1 P}} L d \sigma+\int_{\gamma_{2 P}} L d \sigma \\
& =0+\int_{\gamma_{2 P}} L d \sigma \\
& =L \sigma
\end{aligned}
$$

where $\sigma$ is the length of the arc $\gamma_{2 P}$.

Combining this result with the fact that $A=A_{\gamma_{Q}}-A_{\gamma_{P}}$ yields

$$
A_{\gamma_{Q}}=L \sigma+A_{\gamma_{P}}
$$

We began by claiming that the Prytz planimeter can be used to measure the area of region enclosed by a planar curve by computing $L \sigma$. Here we see that we can use $L \sigma$ to approximate the area if we are willing to live with an error of $A_{\gamma_{P}}$, the (net-signed) area enclosed by the closed curve generated by the knife edge as it follows the planimeter tip and is then rotated into it original position.

\section{Minimizing Error}

As one might have guessed from the earlier numerical examples, one way to minimize the error of this approximation is to use a longer planimeter. In [3] the author states that "if an area whose longest diameter is four inches or less, be measured with a ten inch planimeter, the error is very small and is about equal to the error made in finding the area of an equivalent rectangle by measuring the sides with a scale." Figure 19 shows the situation with a planimeter of length $L=10$ tracing around a square with side length 2.8 (yielding a maximum diameter of just under four). 


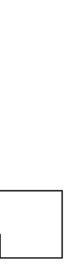

Figure 19: Follow link for animation of $L=10$ planimeter tracing around the square.

If one starts with the planimeter along the positive $y$-axis, Figure 20 shows the initial and final positions of the planimeter. In this case, $s \approx .697$ yielding the estimate $A \approx 6.97$, an error of $11 \%$.

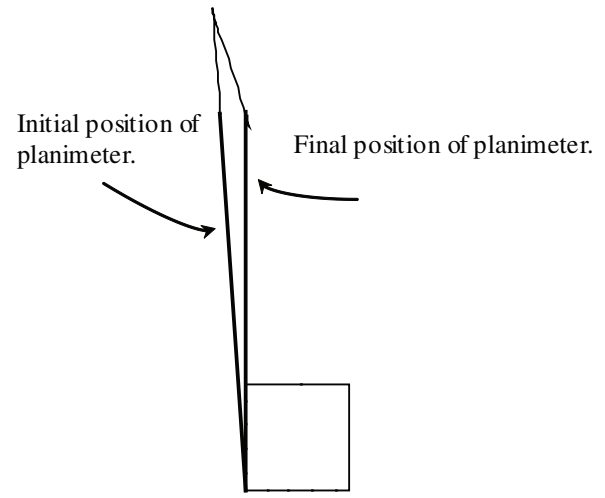

Figure 20: Initial and final positions of $L=10$ planimeter tracing a square of side length 2.8.

Users of Prytz planimeters also originally noticed that the net-signed area $A_{\gamma_{P}}$ tends to be very small when one modifies the tracing of $\gamma_{Q}$ in the following way. Start with the tip of the planimeter near the center of the region whose area you wish to determine. Then, follow a line segment out to $\gamma_{Q}$, around $\gamma_{Q}$, and back to the center. The area enclosed by this new curve is the same as that enclosed by $\gamma_{Q}$ and the curve $\gamma_{P}$ tends to enclose regions of both positive and negative area, thus resulting in a small error. Using the same planimeter and square as above, this procedure is demonstrated in Figure 21. 
Figure 21: Animation of planimeter tracing the square above, this time starting at the center of the square.

Figure 22 shows a possible initial and final position of the planimeter yielding $s \approx .789$ and $A \approx 7.89$, an error of $0.7 \%$.

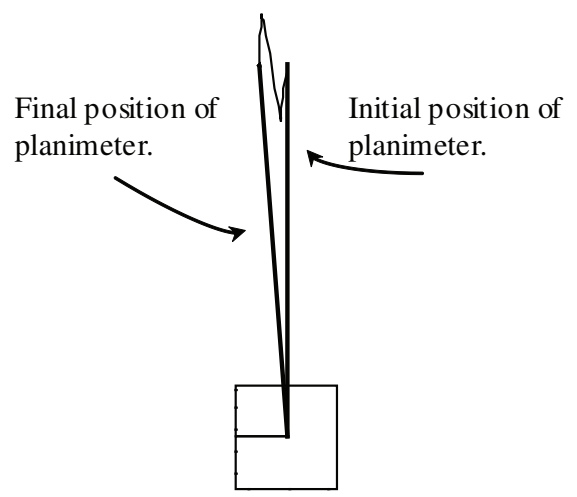

Figure 22: The planimeter begins tracing at the center of the rectangle, proceeds to the left edge, traces the square counter-clockwise, and returns to the center. This path reduces the error in the area estimate.

[4] offers some numerical estimates of the area using this method.

While more accurate planimeters exist, they rely on more precise measuring devices, involve different mathematical computations, and lack the amazing simplicity of Prytz' instrument.

\section{Conclusion}

The examples of Foucault's pendulum and Prytz's planimeter both illustrate how a change in an angle can yield new mathematical information. Once can prove that the Earth rotates and identify one's latitude by observing the motion of the pendulum. One can approximate the area of a region on a map by basically dragging a line segment around the perimeter. While both calculations might appear mysterious, the mathematics which reveal their inner workings is straight-forward and elegant. Ask your teachers for a special course on differential geometry and holonomy! 


\section{Acknowledgements}

I am especially indebted to Robert Foote. Dr. Foote provides a much deeper analysis of the Prytz planimeter in [5]. Dr. Foote also graciously shared his Mathematica code which helped in generating many of the pictures and animations involving the planimeter.

The illustration of the Prytz planimeter is from [2].

The above exposition of Foucault's pendulum using a rectangular basis appeared in [6] and appears in more technical guise in [7].

Special thanks to James Haines, Aaron Montgomery, and Timothy Englund for help and compassion in converting the many figures into a readable format. 


\section{References Cited}

1. Rene Dugas, A History of Mechanics, Central Book Company, Inc., 1955, p382.

2. Olaf Pedersen, "The Prytz Planimeter" in From Ancient Omens to Statistical Mechanics, edited by J.L. Berggren and R.R. Goldstein (University Library, Copenhagen, 1987).

3. A. R. Crathorne: "The Prytz Planimeter", The American Mathematical Monthly 15, pp. 55-57 (1908).

4. George Barnes: "Hatchet or Hacksaw Blade Planimeter", Am. J. Physics 25, pp. 25-29 (1957).

5. Robert L. Foote: "Geometry of the Prytz Planimeter", Reports on Mathematical Physics 42, pp.249-271 (1998).

6. Stuart Boersma: "A Mathematician's Look at Foucault's Pendulum", Math Horizons, pp. 19-21 and 32, February 2005.

7. John Oprea, Differential Geometry and its Applications, Prentice Hall, 1997, pp198-200. 